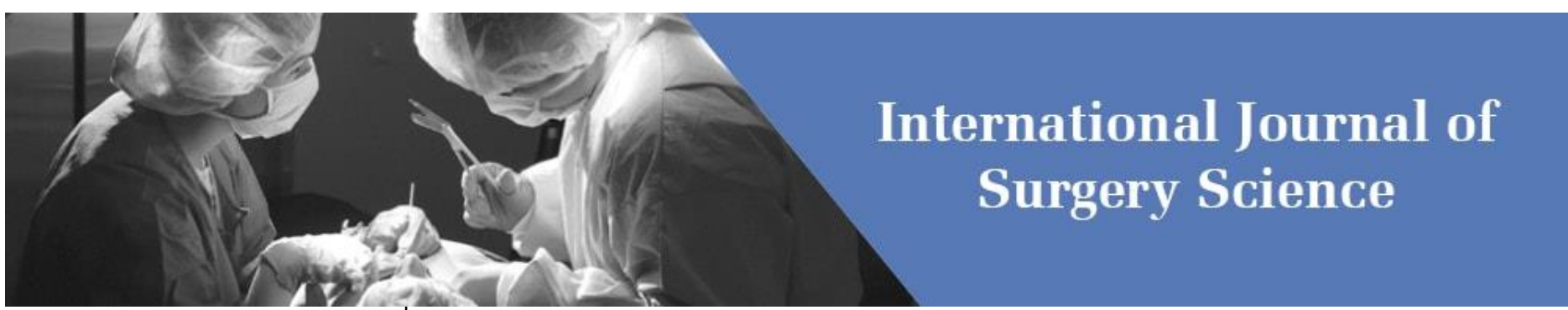

E-ISSN: 2616-3470

P-ISSN: 2616-3462

(C) Surgery Science

www.surgeryscience.com

2020; 4(1): 275-279

Received: 12-11-2019

Accepted: 16-12-2019

Dr. Deepak Naik

Assistant Professor, Department of General Surgery, JSS Medical

College, Jssaher, Mysuru,

Karnataka, India

Dr. Ashwini Kumar Choudhary Junior Resident, Department of General Surgery, JSS Medical College, Jssaher, Mysuru,

Karnataka, India

Dr. Lavanya Raghupathi Junior Resident, Department of General Surgery, JSS Medical College, Jssaher, Mysuru,

Karnataka, India
Corresponding Author: Dr. Ashwini Kumar Choudhary Junior Resident, Department of General Surgery, JSS Medical College, Jssaher, Mysuru,

Karnataka, India

\section{Neutrophils and lymphocytes in various stages of carcinoma breast}

\section{Dr. Deepak Naik, Dr. Ashwini Kumar Choudhary and Dr. Lavanya Raghupathi}

DOI: https://doi.org/10.33545/surgery.2020.v4.i1e.347

\section{Abstract}

Introduction: Neutrophil and lymphocytes are the main inflammatory cells of our body which act as line of defense against various infections. Breast cancer being one of the most common type of cancer in Indian women, is more prevalent in elderly. Its progression and prognosis are influenced by the inflammatory response of the patient in the surrounding tumor microenvironment. Neutrophil-to-lymphocyte ratio (NLR) provides a surrogate marker for prognosis of carcinoma breast.

Aims and objectives: To compare various stages of carcinoma breast with different ranges of NLR and draw a correlation.

Methodology: A descriptive study was done at JSS Hospital over a period of two years for 100 patients presenting with clinically palpable breast nodules which were malignant and results were drawn. All demographic data was summarized as proportion, median, standard deviation and the inferential statistics were obtained using Chi-square and Cramer's V tests.

Results: If NLR is less than median (2.03) majority of the cases of carcinoma breast were found to be Stage I i.e.14.8 percent being less than NLR- 1.5 and 22.2 percent between NLR -1.51 to 2.0 respectively) or Stage I i.e. 55.5 being less than NLR-1.5 and 44.4 percent between NLR 1.51 to 2.0 respectively), however if NLR was more than median the propensity of Stage III carcinoma breast increases progressively.

Conclusion: Increase in neutrophils points towards advanced malignancy as corroborated by higher NLR, thereby making neutrophils and lymphocytes as significant indicators of malignancy in a clinically palpable breast nodule.

Keywords: Neutrophils, lymphocytes, neutrophil lymphocyte ratio (NLR) histopathology, carcinoma breast

\section{Introduction}

Neutrophils are a type of white blood cell that lead the immune system's response. There are four other types of WBC's of which neutrophils are the most abundant, making up 55 to 70 percent. It is one of the first cell types to travel to the site of an infection and help fight infection by ingesting microorganisms and releasing enzymes that kill the microorganisms. Lymphocytes are the immune cells that are made in the bone marrow and are found in the blood and in lymph tissue. The two main types of lymphocytes are B lymphocytes and T lymphocytes of which B lymphocytes are associated with antibodies formation, and T lymphocytes help kill tumor cells thereby controlling immune responses.

Breast cancer being one of the most common type of cancer in Indian women, is more prevalent in elderly. As in other tumours, its development is associated with systemic inflammation which can get modified with tumor growth, invasion, angiogenesis and metastasis. "Inflammatory markers such as c reactive protein (CRP) and interleukin 6 (IL 6) are increased in breast cancer patients" ${ }^{[1]}$. Its progression and prognosis are influenced by the inflammatory response of the patient in the surrounding tumor microenvironment ${ }^{[2]}$. As participants of systemic inflammatory response, neutrophils and lymphocytes are recognized to have a significant role in carcinogenesis and hence tumor progression ${ }^{[3]}$.

Neutrophil to Lymphocyte Ratio (NLR) being parameter of systemic inflammation, is associated with prognosis in various types of cancers including gastrointestinal tract cancers, hepatocellular carcinoma, non-small cell carcinoma and cervical carcinoma ${ }^{[4-8]}$.

Various studies have found that patients with breast cancer exhibit abnormal leukocyte fractions, such as elevated neutrophil count and diminished lymphocyte count, and that the neutrophil-tolymphocyte ratio (NLR) provides a surrogate marker for prognosis and response to treatment of patients after radical surgery. 
Tumor-node-metastasis (TNM) system is a commonly used staging system in clinics rather than prognosis evaluation tool. Accumulating studies show that elevated NLR was associated with high mortality of breast cancer ${ }^{[9,10]}$.

\section{Objectives}

\section{Primary objective}

1. To compare neutrophil to lymphocyte [NLR] ratio in various stages of carcinoma breast

\section{Secondary objective}

1. To compare tumour size ( $\mathrm{T}$ stage) in carcinoma breast to NLR

2. To compare nodal metastasis ( $\mathrm{N}$ stage) in carcinoma breast to NLR

\section{Methodology}

a. Study design: Descriptive Study

b. Study place: JSS Hospital Department of Surgery

c. Study duration: January 2017 to December 2019

d. Sample size: Sample Size is considered as 100

e. Sampling technique and study population: Convenient sample. All consecutive cases coming to surgical department.

f. Inclusion criteria: All proven cases of Carcinoma Breast who have under gone modified radical mastectomy

g. Exclusion criteria

1. Benign breast nodules

2. Patients with bleeding diathesis

3. Patients unwilling for modified radical mastectomy

4. Patients not willing to be part of study

Study assessments of end points: Classify carcinoma breast in various stages after histopathology following modified radical mastectomy and compare NLR in various stages.

\section{Statistical methods applied}

Data analysis

All demographic data is summarized as proportion, mean, standard deviation and tabulated. All the measurements are done using SPSS version 21 and EPI-Info version 7

\section{Descriptive statistics}

The Descriptive procedure displays univariate summary statistics for several variables in a single table and calculates standardized values ( $\mathrm{z}$ scores). Variables can be ordered by the size of their means (in ascending or descending order), alphabetically, or by the order in which the researcher specifies.

\section{Descriptive statistics included}

Median,

Standard deviation,

Frequency and

Percent

\section{Inferential statistics \\ Chi-square test}

The Chi-Square Test procedure tabulates a variable into categories and computes a chi-square statistic. This goodness-offit test compares the observed and expected frequencies in each category to test either that all categories contain the same proportion of values or that each category contains a userspecified proportion of values.

\section{Crosstabs (Cramer's V)}

The Crosstabs procedure forms two-way and multiway tables and provides a variety of tests and measures of association for two-way tables. The structure of the table and whether categories are ordered determine what test or measure to use. Cramer's V test was employed in the present study.

\section{Results}

Neutrophil lymphocyte ratio [NLR] was calculated for all stages and a median of 2.03 was taken for all values, the lowest value being 1.27 and maximum being 3.34. Based on this different categories of NLR were created in this study-

1. Category A having NLR of less than equal to 1.5

2. Category B having NLR of 1.51-2.0

3. Category $\mathrm{C}$ having NLR of $2.01-2.5$

4. Category D having NLR of more than 2.5

Table 1: Tumour stage with NLR

\begin{tabular}{|c|c|c|c|c|c|c|c|}
\hline \multicolumn{8}{|c|}{ Crosstab } \\
\hline & & & \multicolumn{4}{|c|}{ NLR } & \multirow{2}{*}{ Total } \\
\hline & & & $\mathbf{A}$ & B & $\mathrm{C}$ & D & \\
\hline \multirow{8}{*}{ Tumour Stage } & \multirow{2}{*}{$\mathrm{T} 1$} & Count & 6 & 2 & 1 & 5 & 14 \\
\hline & & $\%$ within NLR & $22.2 \%$ & $22.2 \%$ & $6.7 \%$ & $10.2 \%$ & $14.0 \%$ \\
\hline & \multirow{2}{*}{$\mathrm{T} 2$} & Count & 16 & 6 & 12 & 31 & 65 \\
\hline & & $\%$ within NLR & $59.3 \%$ & $66.7 \%$ & $80.0 \%$ & $63.3 \%$ & $65.0 \%$ \\
\hline & \multirow{2}{*}{ T3 } & Count & 5 & 0 & 2 & 11 & 18 \\
\hline & & $\%$ within NLR & $18.5 \%$ & $0.0 \%$ & $13.3 \%$ & $22.4 \%$ & $18.0 \%$ \\
\hline & \multirow{2}{*}{$\mathrm{T} 4$} & Count & 0 & 1 & 0 & 2 & 3 \\
\hline & & $\%$ within NLR & $0.0 \%$ & $11.1 \%$ & $0.0 \%$ & $4.1 \%$ & $3.0 \%$ \\
\hline \multirow{2}{*}{\multicolumn{2}{|c|}{ Total }} & Count & 27 & 9 & 15 & 49 & 100 \\
\hline & & $\%$ within NLR & $100.0 \%$ & $100.0 \%$ & $100.0 \%$ & $100.0 \%$ & $100.0 \%$ \\
\hline
\end{tabular}

On comparing $\mathrm{T}$ Stage (Tumour size) with neutrophil lymphocyte ratio category A i.e. case having NLR less than equal to 1.5 were divided as 22.2 percent in $\mathrm{T} 1,59.3$ percent in $\mathrm{T} 2$ and 18.5 percent in $\mathrm{T} 3$.

Cases having NLR in category B i.e. between 1.51-2.0 were divided as 22.2 percent in $\mathrm{T} 1,66.7$ percent in $\mathrm{T} 2$ and 11.1 percent in $\mathrm{T} 4$.
Cases having NLR in Category $\mathrm{C}$ i.e. between 2.01 and 2.5 were divided as 6.7 percent in T1, 80 percent in $\mathrm{T} 2$ and 13.3 percent in T3.

Cases having NLR in Category D i.e. more than 2.5 were divided as 10.2 percent in T1, 63.3 percent in T2, 22.4 percent in $\mathrm{T} 3$ and 4.1 percent in T4. Maximum cases were staged as T2 while least as T4. 


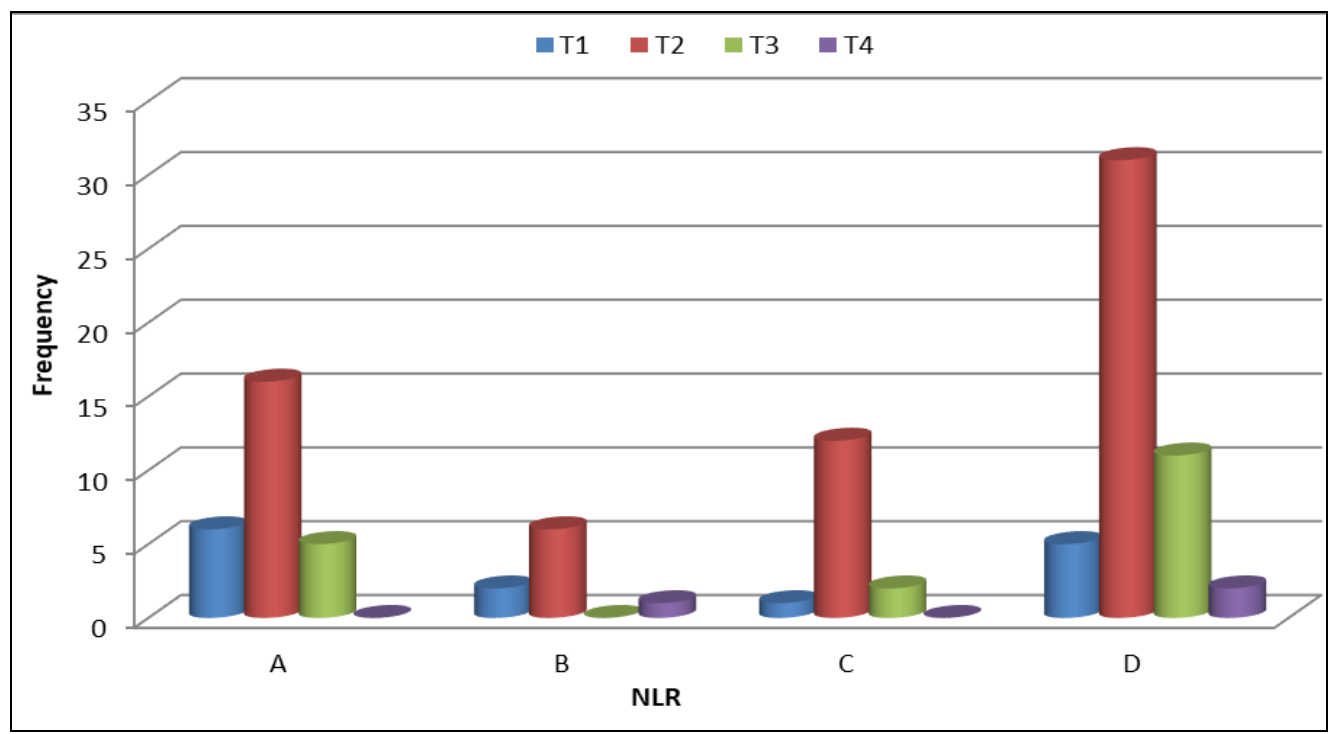

Fig 1: Tumour staging with NLR

Table 2: Nodal Staging Vs NLR

\begin{tabular}{|c|c|c|c|c|c|c|c|}
\hline \multicolumn{8}{|c|}{ Crosstab } \\
\hline & & & \multicolumn{4}{|c|}{ NLR } & \multirow{2}{*}{ Total } \\
\hline & & & $\mathbf{A}$ & B & C & D & \\
\hline \multirow{8}{*}{ NODE } & \multirow{2}{*}{ No } & Count & 14 & 7 & 6 & 18 & 45 \\
\hline & & $\%$ within NLR & $51.9 \%$ & $77.8 \%$ & $40.0 \%$ & $36.7 \%$ & $45.0 \%$ \\
\hline & \multirow{2}{*}{ N1 } & Count & 7 & 0 & 4 & 17 & 28 \\
\hline & & $\%$ within NLR & $25.9 \%$ & $0.0 \%$ & $26.7 \%$ & $34.7 \%$ & $28.0 \%$ \\
\hline & \multirow{2}{*}{$\mathrm{N} 2$} & Count & 1 & 1 & 1 & 4 & 7 \\
\hline & & $\%$ within NLR & $3.7 \%$ & $11.1 \%$ & $6.7 \%$ & $8.2 \%$ & $7.0 \%$ \\
\hline & \multirow{2}{*}{ N3 } & Count & 5 & 1 & 4 & 10 & 20 \\
\hline & & $\%$ within NLR & $18.5 \%$ & $11.1 \%$ & $26.7 \%$ & $16.3 \%$ & $20.0 \%$ \\
\hline \multirow{2}{*}{\multicolumn{2}{|c|}{ Total }} & Count & 27 & 9 & 15 & 49 & 100 \\
\hline & & $\%$ within NLR & $100.0 \%$ & $100.0 \%$ & $100.0 \%$ & $100.0 \%$ & $100.0 \%$ \\
\hline
\end{tabular}

On comparing N Stage (Nodal Metastasis) with neutrophil lymphocyte ratio category A i.e. case having NLR less than equal to 1.5 were divided as 51.9 percent in N0, 25.9 percent in $\mathrm{N} 1,3.7$ percent in N2 and 18.5 percent in N3.

Cases having NLR in category B i.e. between 1.51-2.0 were divided as 77.8 percent in N0, 11.1 percent in $\mathrm{N} 2$ and 11.1 percent in $\mathrm{T} 3$.
Cases having NLR in Category C i.e. between 2.01 and 2.5 were divided as 40.0 percent in N0, 26.7 percent in N1, 6.7 percent in $\mathrm{N} 2$ and 26.7 percent in N3.

Cases having NLR in Category D i.e. more than 2.5 were divided as 36.7 percent in N0, 34.7 percent in N1, 8.2 percent in $\mathrm{N} 2$ and 16.3 percent in N3. Maximum cases were staged as N0 while least as $\mathrm{N} 2$.

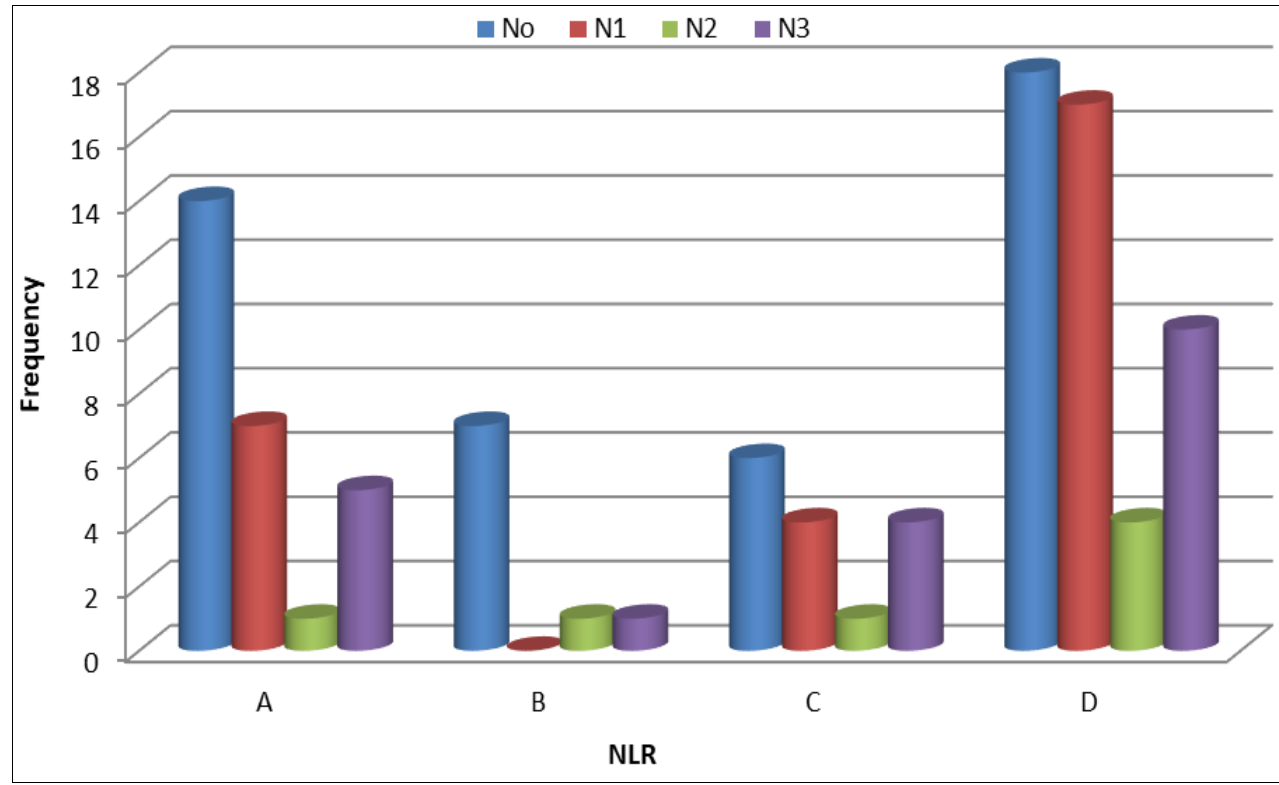

Fig 2: Nodal with NLR 
Table 3: Stages of Carcinoma Breast Vs NLR

\begin{tabular}{|c|c|c|c|c|c|c|c|}
\hline \multicolumn{8}{|c|}{ Crosstab } \\
\hline & & & \multicolumn{4}{|c|}{ NLR } & \multirow[b]{2}{*}{ Total } \\
\hline & & & A & B & $\mathrm{C}$ & $\mathrm{D}$ & \\
\hline \multirow{6}{*}{ Stage } & \multirow{2}{*}{ I } & Count & 4 & 2 & 1 & 4 & 11 \\
\hline & & $\%$ within NLR & $14.8 \%$ & $22.2 \%$ & $6.7 \%$ & $8.2 \%$ & $11.0 \%$ \\
\hline & \multirow{2}{*}{ II } & Count & 15 & 4 & 7 & 24 & 50 \\
\hline & & $\%$ within NLR & $55.6 \%$ & $44.4 \%$ & $46.7 \%$ & $49.0 \%$ & $50.0 \%$ \\
\hline & \multirow{2}{*}{ III } & Count & 8 & 3 & 7 & 21 & 39 \\
\hline & & $\%$ within NLR & $29.6 \%$ & $33.3 \%$ & $46.7 \%$ & $42.9 \%$ & $39.0 \%$ \\
\hline \multirow{2}{*}{\multicolumn{2}{|c|}{ Total }} & Count & 27 & 9 & 15 & 49 & 100 \\
\hline & & $\%$ within NLR & $100.0 \%$ & $100.0 \%$ & $100.0 \%$ & $100.0 \%$ & $100.0 \%$ \\
\hline
\end{tabular}

On comparing various stages of carcinoma breast with neutrophil lymphocyte ratio Category A i.e. case having NLR less than equal to 1.5 were divided as 14.8 percent in Stage I, 55.6 percent in Stage II, 3.7 percent in N2 and 18.5 percent in N3.

Cases having NLR in category B i.e. between 1.51-2.0 were divided as 22.2 percent in Stage I, 44.4 percent in Stage II and 33.3 percent in Stage III.
Cases having NLR in Category C i.e. between 2.01 and 2.5 were divided as 6.7 percent in Stage I, 46.7 percent in Stage II and46.7 percent in Stage III.

Cases having NLR in Category D i.e. more than 2.5 were divided as 8.2 percent in Stage I, 49.0 percent in Stage II and42.9 percent in Stage III. Maximum cases were in staged as Stage 11 while least in Stage I.

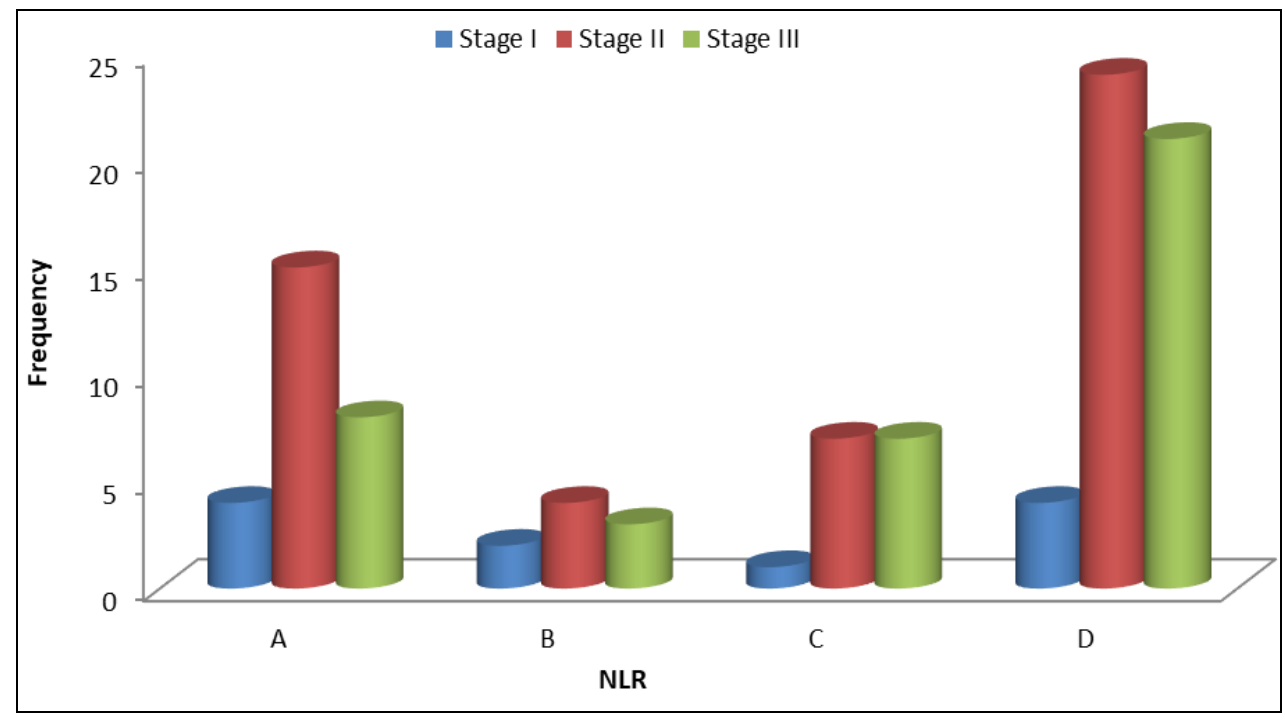

Fig 3: Carcinoma Breast Stage Vs NLR

\section{Discussion}

Inflammation and immunity play an important role in the progression of tumours ${ }^{[11]}$. and also serve as crucial hallmark of the neoplasms.

"Neutrophil-to-lymphocyte ratio (NLR), which has a comprehensive evaluation of the balance between systemic inflammation and immunity, plays a necessary role in prognostic prediction of various malignancies" [12].

NLR is an inexpensive and simple parameter of systemic inflammation. Tumour-infiltrating lymphocytes are front runners in the immune surveillance of tumours, and the predictive and prognostic influence of lymphocyte infiltration and specific subpopulations has been already demonstrated in breast cancer $[13,14]$.

The value of the NLR has been studied in several solid malignancies, and it has been found to be associated with shorter overall survival by former study ${ }^{[15]}$.

"Azab et al. ${ }^{[9]}$ found that patients with breast cancer who had higher NLR showed higher mortality rates compared with those with lower NLR $(P<.001)$, of whom also possessed more severe tumor burden"

"In addition, Dirican A's retrospective study with 1527 breast cancer patients showed that disease-free survival and overall survival were both significantly associated with NLR ${ }^{[16]}$.

The presence of lymphocytes is linked with better chances of survival in patients with cancer ${ }^{[17]}$. In few studies, the presence of different kinds of infiltrating lymphocytes, such as programmed death- 1 positive lymphocytes, and regulatory $\mathrm{T}$ cells is found to be associated with poor survival ${ }^{[18,19]}$.

In our study if NLR was less than median (2.03) than the no. of cases of carcinoma breast having tumour stage $\mathrm{T} 1$ and $\mathrm{T} 2$ were more whereas if NLR increased beyond median value the number of cases of carcinoma breast having tumour stage T3 and $\mathrm{T} 4$ increased.

If NLR was less than median the chances of nodal metastases are less with majority of the cases having no nodal metastasis, when present mostly staged as N1. If NLR is more than median the chances of nodal metastasis are more with majority presenting as either N2 or N3. If NLR is less than median majority of the cases of carcinoma breast were found to be Stage I or Stage II, however if NLR was more than median the propensity of Stage III carcinoma breast increases progressively. NLR has shown in different researches its prognostic relevance in early breast cancer, with higher values associated to poor 
overall and disease free survival. However, only discordant, unsatisfactory and mixed results are available in the metastatic setting.

\section{Conclusion}

- Neutrophils serve as an important inflammatory marker in prognosis of carcinoma breast along with lymphocytes where a higher neutrophil count and lower lymphocytes point towards advance disease.

- Increase in NLR signifies increase in size of the breast tumour corresponding as higher $\mathrm{T}$ stage.

- Increase in NLR signifies nodal metastasis.

- Increase in NLR signifies advanced carcinoma breast corresponding to a higher overall stage.

\section{Reference}

1. Balta S, Demirkol S, Kucuk U, Sarlak H, Kurt O, Arslan Z. Neutrophil to lymphocyte ratio may predict mortality in breast cancer patients. Journal of breast cancer. 2013; 16(3):354-5.

2. Hanahan D, Weinberg RA. Hallmarks of cancer: the next generation. Cell. 2011; 144:646-674

3. Lal I, Dittus K, Holmes CE. Platelets coagulation and fibrinolysis in breast cancer progression. Breast Cancer Res 2013; 15:207-218.

4. Guthrie GJ, Charles KA, Roxburgh CS, Horgan PG, McMillan DC, Clarke SJ. The systemic inflammation-based neutrophil-lymphocyte ratio: experience in patients with cancer. Critical reviews in oncology/hematology. 2013; 88(1):218-30.

5. Walsh SR, Cook EJ, Goulder F, Justin TA, Keeling NJ. Neutrophil- lymphocyte ratio as a prognostic factor in colorectal cancer. Journal of surgical oncology. 2005; 91(3):181-4.

6. Unal D, Eroglu C, Kurtul N, Oguz A, Tasdemir A. Are neutrophil/lymphocyte and platelet/lymphocyte rates in patients with non-small cell lung cancer associated with treatment response and prognosis?. Asian Pacific Journal of Cancer Prevention. 2013; 14(9):5237-42.

7. Salim DK, Mutlu H, Eryılmaz MK, Salim O, Musri FY, Tural D et al. Neutrophil to lymphocyte ratio is an independent prognostic factor in patients with recurrent or metastatic head and neck squamous cell cancer. Molecular and clinical oncology. 2015; 3(4):839-42.

8. Kemal Y, Yucel I, Ekiz K, Demirag G, Yilmaz B, Teker F, et al. Elevated serum neutrophil to lymphocyte and platelet to lymphocyte ratios could be useful in lung cancer diagnosis. Asian Pac J Cancer Prev. 2014; 15(6):2651-4.

9. Azab B, Bhatt VR, Phookan J et al. Usefulness of the neutrophil-tolymphocyte ratio in predicting short- and longterm mortality in breast cancer patients. Ann Surg Oncol 2012; 19:217-24.

10. Azab B, Shah N, Radbel J, et al. Pretreatment neutrophil/lymphocyte ratio is superior to platelet/lymphocyte ratio as a predictor of long-term mortality in breast cancer patients. Med Oncol (Northwood, London, England). 2013; 30:432.

11. Candido J, Hagemann T. Cancer-related inflammation. J ClinImmunol. 2013; 33(1):S79-84.

12. Gregory AD, Houghton AM. Tumor-associated neutrophils: new targets for cancer therapy. Cancer Res. 2011; 71:24116.

13. Savas $\mathrm{P}$ et al. Clinical relevance of host immunity in breast cancer: from TILs to the clinic. Nat. Rev. Clin. Oncol.
2016; 13:228-241

14. García-Martínez E et al. Tumour-infiltrating immune cell profiles and their change after neoadjuvant chemotherapy predict response and prognosis of breast cancer. Breast Cancer Res. 2014; 16:488, https://doi.org/10.1186/s13058014-0488-5

15. Templeton AJ, McNamara MG, Seruga B et al. Prognostic role of neutrophil-to-lymphocyte ratio in solid tumors: a systematic review and meta-analysis. J Natl Cancer Inst. 2014; 106:u124.

16. Dirican A, Kucukzeybek BB, Alacacioglu A et al. Do the derived neutrophil to lymphocyte ratio and the neutrophil to lymphocyte ratio predict prognosis in breast cancer? Int $\mathrm{J}$ ClinOncol. 2015; 20:70-81.

17. Adams S, Gray RJ, Demaria S, Goldstein L, Perez EA, Shulman LN et al. Prognostic value of tumor-infiltrating lymphocytes in triple-negative breast cancers from two phase III randomized adjuvant breast cancer trials: ECOG 2197 and ECOG 1199. J Clin Oncol. 2014; 32:2959-2966.

18. Muenst S, Soysal SD, Gao F, Obermann EC, Oertli D, Gillanders WE et al. The presence of programmed death 1 (PD-1)-positive tumor-infiltrating lymphocytes is associated with poor prognosis in human breast cancer. Breast Cancer Res Treat. 2013; 139:667-676.

19. Tao H, Mimura Y, Aoe K, Kobayashi S, Yamamoto H, Matsuda E et al. Prognostic potential of FOXP3 expression in non-small cell lung cancer cells combined with tumorinfiltrating regulatory $\mathrm{T}$ cells. Lung Cancer. 2012; 75:95101. 